\title{
Transcriptome analysis of Chelidonium majus elaiosomes and seeds provide insights into fatty acid biosynthesis
}

\author{
Jiayue Wu ${ }^{1}$, Linlin Peng ${ }^{1}$, Shubin Dong ${ }^{2}$, Xiaofei Xia ${ }^{3}$, Liangcheng Zhao ${ }^{\text {Corresp. } 1,4}$ \\ ${ }^{1}$ College of Nature Conservation, Beijing Forestry University, Beijing, China \\ 2 College of Biological Sciences and Technology, Beijing Forestry University, Beijing, China \\ 3 Beijing Museum of Natural History, Beijing, China \\ 4 Museum of Beijing Forestry University, Beijing Forestry University, Beijing, China \\ Corresponding Author: Liangcheng Zhao \\ Email address: Iczhao@bjfu.edu.cn
}

Background. Elaiosomes are specialized fleshy and edible seed appendages dispersed by ants. Lipids are the primary components of elaiosomes. Chelidonium majus is a well-known plant, the seeds of which are dispersed by ants. Previous studies have identified the presence of primary fatty acids in its elaiosomes and seeds. However, the molecular mechanisms underlying fatty acid biosynthesis in elaiosomes remain unknown.

Methods. In order to gain a comprehensive transcriptional profile of the elaiosomes and seeds of $C$. majus, and understand the expression patterns of genes associated with fatty acid biosynthesis, four different developmental stages, including the flower-bud (Ch01), flowering (Ch02), young seed (Ch03), and mature seed (Ch04) stages, were chosen to perform whole-transcriptome profiling through the RNAseq technology (Illumina NGS sequencing).

Results. A total of 63,064 unigenes were generated from 12 libraries. Of these, 7,323, 258, and 11,540 unigenes were annotated with 25 clusters of orthologous groups (COGs), 43 gene ontology (GO) terms, and 373 Kyoto Encyclopedia of Genes and Genomes (KEGG) pathways, respectively. In addition, 322 genes were involved in lipid transport and metabolism, and 508 genes were involved in the lipid metabolism pathways. Forty-one significantly differentially expressed genes (DEGs) involved in the lipid metabolism pathways were identified, most of which were upregulated in Ch03 compared to Ch02, indicating that fatty acid biosynthesis primarily occurs during the flowering to the young seed stages. Of the DEGs, acyl-ACP thioesterases, acyl carrier protein desaturase (DESA1), and malonyl CoA-ACP transacylase were involved in palmitic acid synthesis; stearoyl-CoA desaturase and DESA1 were involved in oleic acid synthesis, and acyl-lipid omega-6 desaturase was involved in linoleic acid synthesis. 


\section{Transcriptome analysis of Chelidonium majus \\ 2 elaiosomes and seeds provide insights into fatty acid \\ 3 biosynthesis}

5 Jiayue $\mathrm{Wu}^{1}$, Linlin Peng ${ }^{1}$, Shubin Dong ${ }^{2}$, Xiaofei Xia ${ }^{3}$, Liangcheng Zhao ${ }^{1,4^{*}}$

$7{ }^{1}$ College of Nature Conservation, Beijing Forestry University, Beijing, China

$8{ }^{2}$ College of Biological Sciences and Technology, Beijing Forestry University, Beijing, China

$9 \quad{ }^{3}$ Beijing Museum of Natural History, Beijing, China

$10{ }^{4}$ Museum of Beijing Forestry University, Beijing, China

11

12

Corresponding Author:

Liangcheng Zhao

No. 35 East Qinghua Road, Haidian District, Beijing, 100083, China

Email address: Iczhao@bjfu.edu.cn

\section{ABSTRACT}

Background. Elaiosomes are specialized fleshy and edible seed appendages dispersed by ants. Lipids are the primary components of elaiosomes. Chelidonium majus is a well-known plant, the seeds of which are dispersed by ants. Previous studies have identified the presence of primary fatty acids in its elaiosomes and seeds. However, the molecular mechanisms underlying fatty acid biosynthesis in elaiosomes remain unknown.

Methods. In order to gain a comprehensive transcriptional profile of the elaiosomes and seeds of C. majus, and understand the expression patterns of genes associated with fatty acid biosynthesis, 
27 four different developmental stages, including the flower-bud (Ch01), flowering (Ch02), young 28 seed (Ch03), and mature seed (Ch04) stages, were chosen to perform whole-transcriptome profiling through the RNA-seq technology (Illumina NGS sequencing).

30

Results. A total of 63,064 unigenes were generated from 12 libraries. Of these, 7,323, 258, and 11,540 unigenes were annotated with 25 clusters of orthologous groups (COGs), 43 gene ontology (GO) terms, and 373 Kyoto Encyclopedia of Genes and Genomes (KEGG) pathways, respectively. In addition, 322 genes were involved in lipid transport and metabolism, and 508 genes were involved in the lipid metabolism pathways. Forty-one significantly differentially expressed genes (DEGs) involved in the lipid metabolism pathways were identified, most of which were upregulated in Ch03 compared to Ch02, indicating that fatty acid biosynthesis primarily occurs during the flowering to the young seed stages. Of the DEGs, acyl-ACP thioesterases, acyl carrier protein desaturase (DESA1), and malonyl CoA-ACP transacylase were involved in palmitic acid synthesis; stearoyl-CoA desaturase and DESA1 were involved in oleic acid synthesis, and acyl-lipid omega- 6 desaturase was involved in linoleic acid synthesis.

\section{INTRODUCTION}

Elaiosomes are highly specialized nutrient-rich structures that are attached to the seeds of many plant species. The term elaiosome was introduced for the first time by Sernander (1906) to refer to all fleshy and edible appendages of the diaspore dispersed by ants. Seed dispersal by ants is known as myrmecochory, a worldwide phenomenon found in a diverse range of habitats, including arid, tropical, and temperate regions (Van der Pijl, 1982; Werker, 1997; Giladi, 2006). A recent study estimated that more than 11,000 species in 334 genera (about $2.5 \%$ of all genera) and 77 families (around 17\% of all families) of angiosperm plants could be dispersed via myrmecochory (Lengyel et al., 2010).

Myrmecochory is regarded as a mutualistic relationship that is beneficial for both plants and ants. Ants typically collect and transfer whole diaspores (seed + elaiosome) to their nests, wherein the elaiosomes are consumed and the seeds are discarded unharmed within or outside the nests, which are in turn generally regarded to provide optimal germinating conditions (Beattie, 1985; Hughes \& Westoby, 1990; Giladi, 2006). The elaiosomes can be fed to the larvae, 
56

57

58

59

60

61

62

63

64

65

66

67

68

69

70

71

72

73

74

75

76

77

78

79

80

81

82

83

84

85

which in turn promotes ant colony reproduction, fitness, and gyne production (Morales \& Heithaus, 1998; Bono \& Heithaus, 2002; Fischer et al., 2005; Gammans, Bullock \& Schönrogge, 2005). Therefore, elaiosomes are key structures that increase the attractiveness of diaspores to ants (Reifenrath, Becker \& Poethke, 2012). Elaiosomes develop from seed tissues (chalaza, funiculus, hilum, and raphe-antiraphe) through various mechanisms, but appear to have the same main function of attracting ants (Lisci, Bianchini \& Pacini, 1996; Morrone, Vega \& Maier, 2000; Ciccarelli et al., 2005; Yang et al., 2015). Hence, elaiosomes are considered suitable examples for studying convergent evolution in flowering plants (Lengyel et al., 2010).

Many studies have described several factors that likely determine the efficiency of myrmecochory. The sizes of seeds and elaiosomes, the elaiosome size/seed size ratio, and their physical surfaces influence the ability of ants to disperse the diaspores (Oostermeijer, 1989; Hughes \& Westoby, 1992; Mark \& Olesen, 1996). In addition to the morphological and structural characteristics of diaspores, chemical compounds present in the elaiosomes are additionally regarded as crucial factors that influence the removal of diaspores, considering that elaiosomes are beneficial for diaspore transport and food acquisition in ants (Hughes, Westoby \& Jurado, 1994; Fischer et al., 2008; Reifenrath, Becker \& Poethke, 2012; Chen et al., 2016). Although protein and starch are also present in elaiosomes, lipids generally constitute the major class of compounds in elaiosomes. Based on the prominent hypothesis that certain fatty acids induce the collection of diaspores by ants, most studies have focused on the fatty acids of the elaisomes and compared their compositions with those of seeds or even insects (Marshall, Beattie \& Bollenbacher, 1979; Soukup \& Holman, 1987; Kusmenoglu, Rockwood \& Gretz,1989; Lanza, Schmitt \& Awad, 1992; Hughes, Westoby \& Jurado, 1994; Morrone, Vega \& Maier, 2000; Boulay, Coll-Toledano \& Cerdá, 2006). A more recent chemical analysis of the diaspores of 13 central European ant-dispersed plant species from seven families revealed that elaiosomes have a more homogeneous fatty acid composition, with oleic acid as the most abundant fatty acid component, followed by palmitic acid and linoleic acid (Fischer et al., 2008).

Chelidonium majus L., commonly known as greater celandine, is the only species of the genus Chelidonium in the Papaveraceae family. C. majus is an herbaceous perennial plant (Fig. 1A) that is widely distributed in Europe and Asia. C. majus is rich in various types of isoquinoline alkaloids and is widely used against various diseases, such as ulcer, gastric cancer, 
86

87

88

89

90

91

92

93

94

95

96

97

98

99

100

101

102

103

104

105

106

107

108

109

110

111

112

113

114

oral infection, liver disorders, chronic bronchitis, asthma, and general pains, in European countries and Chinese traditional medicine (Arora \& Sharma, 2013; Maji \& Banerji, 2015). C. majus has attracted research attention not only for its medicinal value, but also for its wellknown role in ant-dispersal (Fig. 1I). As a typical myrmecochorus plant, C. majus has been investigated in several studies on ant-seed interactions (Gorb \& Gorb, 2000; Fischer et al., 2008; Servigne \& Detrain, 2008; Reifenrath, Becker \& Poethke, 2012). In terms of its reproductive features, $C$. majus is characterized by large yellow petals, long cylindrical capsules, and numerous small seeds (ca. 1.1-1.5 mm in length) with conspicuous coronal elaiosomes (Figs. 1C1F). The elaiosomes (ca. 0.8-1.1 mm in length) are white and transparent, which contrasts with the glossy black color of the seeds (Figs. 1F,1G).

The structure and development of elaiosomes in C. majus were briefly described by Lisci et al. (1996), who showed that the elaiosomes of C. majus arise from the cells of the raphe and form a ventral excrescence along the seed from the hilum and adjacent part of the micropyle to the chalazal area. Recently, one of the authors of this paper (Liangcheng Zhao) conducted a detailed study on the complete ontogeny of $C$. majus elaiosomes and seeds using standard techniques for plant anatomy and histochemistry (see Yang et al., 2015). Different development stages have been characterized based on distinct morphological and anatomical features that can be used in genetic studies. Results indicated that the meristem of C. majus elaiosome is formed by periclinal divisions of the outmost layer cells of raphe in flower-bud stage (prior to fertilization); after flowering stage (post-pollination) mitotic activity ceases, and the elaiosome develops solely through very active cell growth. The mature elaiosome of $C$. majus is composed of two cell types, small basal cells and very elongated outer cells. Both cell types, especially the outer cells, contain a large amount of lipids (Fig. 1H), as verified by the red-stained oil droplets with Sudan III. In contrast, the seed primarily consists of the endosperm, which contains numerous protein granules (Fig.1H). Reifenrath, Becker \& Poethke (2012) performed quantitative analysis of the elaiosomes by gas chromatography and provided a detailed fatty acid composition of the $C$. majus elaiosomes. The most abundant fatty acid was determined to be linoleic acid, followed by palmitic acid, and then oleic acid; these three fatty acids comprised of nearly $96.5 \%$ of the total fatty acid content of the elaiosomes. 
115

116

117

118

119

120

121

122

123

124

125

126

127

128

129

130

131

132

133

134

135

136

137

138

139

140

141

142

143

144

Although various studies have investigated the lipid composition of elaiosomes and seeds in different plant species and identified the main fatty acids, the regulatory mechanisms related to the biosynthesis of these fatty acids remain unknown. To understand the expression patterns of genes associated with fatty acid biosynthesis in elaisomes and seeds, detailed genome information is essential and indispensable. In the present study, RNA-seq technology was employed to obtain a comprehensive overview of gene expression related to fatty acid biosynthesis in $C$. majus elaiosomes and seeds for the first time. In recent years, next-generation sequencing (NGS), also known as high-throughput sequencing, has emerged as a powerful tool for profiling the whole transcriptome from non-model plants that do not have available reference genomes (Unamba, Nag \& Sharma, 2015). In the present study, we performed wholetranscriptome profiling by NGS to capture the genomic landscape of $C$. majus elaiosomes and seeds. The analysis not only provided important insights into fatty acid biosynthesis in C. majus elaiosomes and seeds, but also served as a genomic resource for future evolutionary studies that focus on the convergent evolution of myrmecochorous plants.

\section{MATERIALS \& METHODS}

\section{Plant Materials and Treatments}

The sampled plants of Chelidonium majus were grown in Beijing Badaling National Forest Park, China, under natural conditions (Fig. 1A). To gain a comprehensive transcriptional profile of its elaiosomes and seeds, sample collection at the optimal developmental stage is crucial. Based on previous observations of C. majus elaiosome and seed development, four different developmental stages were defined (Yang et al., 2015). In the present study, we chose these four developmental stages, namely flower-bud stage (pre-fertilization) (Ch01) (Fig. 1B), flowering stage (post-pollination) (Ch02) (Fig. 1C), young seed stage (Ch03) (Fig. 1E), and mature seed stage (Ch04) (Fig. 1F) for comparative transcriptome analysis to explore gene expression during fatty acid biosynthesis in the elaisomes and seeds of $C$. majus. Corresponding to the four stages, flower buds (with bracts, petals, and stamens removed), fertilized ovaries, young seeds (testa white to brown), and mature seeds (testa black and hard) were collected as RNA-seq materials. They were frozen in liquid nitrogen and stored at $-80{ }^{\circ} \mathrm{C}$ until further use. Three samples were collected as biological repeats for each developmental stage. 


\section{RNA Isolation and Sequencing}

146 To obtain transcriptome expression profiles, RNA-seq transcriptome libraries were prepared

147 using the TruSeqTM RNA sample preparation Kit from Illumina (San Diego, CA, USA) and 5

$148 \mu \mathrm{g}$ of total RNA. First, messenger RNA was isolated through the polyA selection method using

149 oligo (dT) beads and fragmented in a fragmentation buffer. Next, double-stranded cDNA was

150 synthesized using a SuperScript double-stranded cDNA synthesis kit (Invitrogen, CA, USA) and

151 random hexamer primers (Illumina). Then, the synthesized cDNA was subjected to end-repair,

152 phosphorylation, and "A" base addition according to Illumina's library construction protocol.

153 Libraries were size selected for cDNA target fragments of 200-300 bp on a 2\% Low Range Ultra

154 Agarose gel followed by PCR (polymerase chain reaction) amplification using Phusion DNA

155 polymerase (NEB) for 15 cycles. After quantification using TBS380, the paired-end RNA-seq

156 sequencing library was sequenced using Illumina HiSeq 4000 ( $2 \times 150$ bp read length). RNA

157 samples were named Ch01a/b/c (flower-bud stage), Ch02a/b/c (flowering stage), Ch03a/b/c

158 (young seed stage), and Ch04a/b/c (mature seed stage). Twelve cDNA libraries were then

159 constructed and sequenced using the Illumina deep sequencing platform.

160

161

162

163

164

165

166

167

168

169

170

171

172

173

174

175

\section{De novo assembly, Annotation, Differential expression analysis}

The raw paired-end reads were trimmed and quality controlled by SeqPrep and Sickle with default parameters. Then, clean data were used to do de novo assembly with Trinity (Grabherr et al., 2011). All of the assembled transcripts were searched against the NR, COG, and KEGG databases using BLASTX to identify the proteins that had the highest sequence similarity to the given transcripts to retrieve their function annotations; a typical cut-off E-value of less than $1.0 \times 10^{-5}$ was set. The BLAST2GO program was used to get GO annotations of uniquely assembled transcripts for describing biological processes, molecular functions, and cellular components (Conesa et al., 2005). COG is an early database that is expected to be a useful platform for the functional annotation of newly sequenced genomes (Tatusov et al., 2003). The KEGG database is the primary public repository devoted specifically to pathways. Metabolic pathway analysis was performed using KEGG (Kanehisa \& Goto, 2000), and KEGG classifications facilitate the exploration of categories of genes with specific functions. To identify DEGs between different samples, the expression level of each transcript was calculated according to the fragments per kilobase of the exon per million mapped reads (FPKM) method. RSEM (RNA-Seq by Expectation-Maximization) was used to quantify gene and isoform 
176 abundances (Li \& Dewey, 2011). The R statistical package software, EdgeR (Empirical analysis 177 of Digital Gene Expression in R), was utilized for the differential expression analysis (Robinson, 178 McCarthy \& Smyth, 2010). In addition, functional-enrichment analysis, including GO and 179 KEGG, were performed to identify which DEGs were significantly enriched in GO terms and 180 metabolic pathways at a Bonferroni-corrected P-value $\leq 0.05$ compared with the whole181 transcriptome background. GO functional enrichment and KEGG pathway analysis were carried 182 out by Goatools and KOBAS (Xie et al., 2011).

\section{Quantitative Real-time PCR (qRT-PCR) analysis}

For each sample, $1 \mu \mathrm{g}$ of total RNA was used for cDNA synthesis using the Plant RNA 9600 plus Fluorescence quantitative PCR instrument (Hangzhou, China). Each sample contains three independent biological replicates.

\section{RESULTS AND DISCUSSION}

193

194

195

196

197

198

199

200

201

202

203

\section{Transcriptome sequencing and de novo assembly}

In this study, a total of 12 libraries were sequenced. After removing the low-quality reads and adaptor sequences, the remaining clean data comprised of approximately $61 \mathrm{~Gb}$ of sequence data (171 million reads from the flower-bud stage (Ch01); 141 million reads from flowering stage (Ch02); 148 million reads from young seed stage (Ch03); and 154 million reads from mature seed stage (Ch04)). The results are shown in Table 1. De novo assembly of the clean reads was performed using Trinity to generate a reliable integrated reference sequence database (File S1). Some reads comprise of a contig, and some contigs comprise of a unigene. We obtained a total of 63,064 unigenes (File S2) for all studied samples; these unigenes had an average length and N50 value of $1114.56 \mathrm{bp}$ and $1565 \mathrm{bp}$, respectively. After sorting the assembled unigenes from large to small, the N50 value was calculated as half of the total length of the unigenes. The size 
204 distribution of the assembled transcripts is shown in Figure 2. As shown in Table 1 and Figure 2, 205 the sequencing data were of sufficiently high quality for subsequent analyses. Therefore, gene 206 expression data from C. majus elaisomes and seeds were reliable.

\section{Functional annotation and classification of unigenes}

208 To verify the potential functions of the 63,064 assembled unigenes, we performed sequence 209 similarity BLASTx searches against the NR (NCBI protein nonredundant), COG (Clusters of 210 Orthologous Groups), GO (Gene Ontology), and KEGG (Kyoto Encyclopedia of Genes and

211 Genomes) protein databases with E-values $<1 \mathrm{e}^{-5}$. After alignment, gene annotation, functional 212 classification, and metabolic pathway assignment, a total of 30,143 (47.80\%) of the unigenes 213 were successfully annotated (File S3), which indicated that their functions were relatively 214 conserved and diverse.

\section{Characterization of unigene sequencing}

216 Among all the 30,143 annotated unigenes, 29,352 (97.38\%) were mapped to the NR database. In 217 addition, the distribution of E-values of the hits in the NR database indicated that 20,378 218 unigenes, representing more than half of the mapped sequences (69.43\%), showed significant 219 homology $\left(\right.$ E-value $<1.0 \times 10^{-30}$ ), while 8,973 unigenes (about 30.57\%) had E-values ranging 220 from $1.0 \times 10^{-30}$ to $1.0 \times 10^{-5}$ (Fig. 3, File S3). Smaller E-values distribution (Fig. 3A) indicated a 221 higher reliability of hits. The similarity distribution showed that the majority of the mapped 22226,160 unigenes $(89.13 \%)$ had similarity values $>60 \%$, whereas only $3,192(10.87 \%)$ had 223 similarity values $<40 \%$ (Fig. 3B). To identify species specificity, individual unigenes were 224 annotated based on the highest BLAST scores against the NR database. Among flowering plants, 225 6,666 (22.71\%) unigenes showed significant homology to Vitis vinifera, followed by Theobroma 226 cacao (1,983 unigenes, 6.76\%), Phoenix dactylifera (1,230 unigenes, 4.19\%), Populus

227 trichocarpa (1,224 unigenes, 4.17\%), Prunus persica (1,090 unigenes, 3.71\%), and Jatropha 228 curcas (1,065 unigenes, 3.63\%) (Fig. 3C). A total of 2,980 unigenes, representing around $22910.15 \%$ of all unigenes, did not match the NR database, and thus provided novel genomic 230 information on C. majus. Some of these unigenes are likely to be untranslated regions (UTRs) or 231 non-coding RNAs.

\section{Functional classification by Gene Ontology}


233 By conducting GO functional classification, a total of 258 unigenes were classified into one or 234 more terms and divided into 43 functional groups, belonging to three major categories (Fig. 4). 235 In the biological process category, the majority of the assigned proteins were involved in 236 metabolic processes (177 unigenes, 68.60\%), followed by genes involved in cellular processes 237 (169 unigenes, 65.50\%) and single-organism processes (156 unigenes, 60.47\%). Under the 238 molecular function category, the mapped unigenes were categorized into nine groups, out of 239 which genes were predominantly associated with catalytic activity (165 unigenes, 63.95\%) and 240 binding (123 unigenes, 47.67\%), which exceeded the total number of the other terms. The 241 cellular component category was divided into 14 groups, in which the largest assignments were 242 found to be in the cell and cell part. There were 164 unigenes in the cell and cell part (63.57\%), 243 followed by organelles (115 unigenes, 44.57\%), membrane (52 unigenes, 20.16\%), and 244 membrane parts (36 unigenes, 13.95\%). The above results suggested that the development of 245 elaisomes and seeds in C. majus are closely associated with enzyme-catalyzed reactions, cell 246 structure formation, and metabolic processes.

\section{Functional classification by Clusters of Orthologous Groups}

248 To predict the potential function of genes and evaluate the completeness of the transcriptome 249 libraries, all assembled unigenes were searched against the COG database. A total of 7,323 250 mapped unique sequences were clustered into 25 functional categories (Fig. 5). Among them, the 251 largest category was 'general functions prediction only' $(917,12.52 \%)$, followed by 'signal 252 transduction mechanisms' (798, 10.90\%), 'posttranslational modification, protein turnover, and 253 chaperones' (727, 9.93\%), and 'translation, ribosomal structure, and biogenesis' (596, 8.14\%). 254 Thus, we inferred that these genes play an important role in ribosome formation, protein 255 synthesis, and signal transduction during the development of elaisomes and seeds in C. majus. 256 Notably, 322 unigenes (4.40\%) were classified under the 'lipid transport and metabolism' 257 category, which could provide useful information for further studies on lipid metabolism in $C$. 258 majus elaisomes and seeds.

\section{Biological pathways identification by KEGG}

260 To identify the biological pathways that were actively involved in lipids accumulation in $C$.

261 majus elaisomes and seeds, all annotated unigenes were mapped to the reference pathways in the 262 KEGG. The analysis identified a total of 11,540 unigenes that were assigned to five clusters and 
263 distributed across 373 KEGG pathways (Fig. 6). Most of the unigenes were grouped under the 264 metabolism cluster (A). Within this cluster, the majority of unigenes were assigned under the 265 "global and overview maps pathway" (2,736, 23.71\%), followed by "carbohydrate metabolism" 266 (1022, 8.85\%), “amino acid metabolism" (800, 6.93\%), and "energy metabolism" (590, 5.11\%) 267 categories. The relative abundance of unigenes involved in 'amino acid metabolism' pathways is 268 probably related to the biosynthesis of massive storage protein in the C. majus endosperm.

269 Notably, in the "energy metabolism" cluster, some pathways were found to be closely related to 270 the lipid metabolism. A total of 508 unigenes, including glycerophospholipid metabolism (205, $27140.35 \%)$, glycerolipid metabolism (108, 21.26\%), fatty acid degradation (100, 19.69\%), 272 biosynthesis of unsaturated fatty acids $(93,18.31 \%)$, fatty acid elongation $(75,14.76 \%)$, fatty 273 acid biosynthesis (74, 14.57\%), ether lipid metabolism (68, 13.39\%), sphingolipid metabolism $274(54,10.63 \%)$, linoleic acid metabolism (44, 8.66\%), steroid biosynthesis $(42,8.27 \%)$, 275 arachidonic acid metabolism $(21,4.13 \%)$, steroid hormone biosynthesis $(16,3.15 \%)$, synthesis 276 and degradation of ketone bodies (12, 2.36\%), and primary bile acid biosynthesis $(5,0.98 \%)$ 277 pathways were involved in these pathways (Fig. 7).

\section{Analysis of DEGs at four developmental stages}

279 Analysis of the fatty acid compositions of C. majus seeds and elaiosomes showed that the 280 elaiosome-bearing seeds predominantly contained the mono-unsaturated fatty acid, linoleic acid 281 (C18:2) (77.9\%), followed by the mono-unsaturated fatty acid, oleic acid (C18:1) (16\%) 282 (Reifenrath, Becker \& Poethke, 2012). Furthermore, saturated fatty acids accounted for only six 283 percent of the composition, and most of the saturated fatty acids were identified as palmitic acid 284 (C18:1) (3.5\%). However, in only elaiosomes, the most abundant fatty acid also was linoleic acid 285 (37.2\%), followed by palmitic acid (34.8\%), and oleic acid (24.5\%). Previous developmental and 286 histochemical observations (Yang et al., 2015) indicated that the elaiosome meristem of C. majus was formed during the flower-bud stage (Ch01), while elaiosome cell growth and enlargement primarily occurred from the flowering stage (Ch02) to the young seed stage (Ch03); the maturation of the seed and elaiosome occurred from the young seed stage to the mature seed stage (Ch04). Therefore, to obtain insight into the regulation of the biosynthesis of saturated fatty acids and unsaturated fatty acids in elaiosomes and seeds, we analyzed the differentially

292 expressed genes (DEGs) of the lipid metabolism pathways in Ch02 compared to Ch01, Ch03 293 compared to Ch02, and Ch04 compared to Ch03. 


\section{Identification of unigenes related to fatty acid biosynthesis}

295 From the fatty acids biosynthesis pathway (Path: ko00061) and the biosynthesis pathway of 296 unsaturated fatty acids (Path: ko01040) at the four developmental stages of C. majus elaisomes 297 and seeds (File S4), we identified 41 significantly DEGs. These DEGs are shown in File S5 and 298 the corresponging genetic heat map is shown in Figure 8. Four unigenes encoding the enzymes 299 malonyl CoA-ACP transacylase $(f a b G)$ (Toomey \& Wakil, 1966), acyl-CoA oxidase $(A C O X 1)$ 300 (Oaxaca-Castillo et al., 2007), acyl carrier protein desaturase (DESA1) (Kachroo et al., 2007), 301 and $\beta$-ketoacyl-acyl carrier protein synthase II ( $f a b F)$ (Magnuson, Carey \& Cronan, 1995) were

302

303

304

305

306

307

308

309

310

311

312

313

314

315

316

317 318

319

320

321

322

323

324 found to be upregulated in Ch02 compared to Ch01. By contrast, 24 unigenes encoding the enzymes acetyl-coA carboxylase (Accase) (Bilder et al., 2006), acyl coenzyme A synthetase $(A C S L)$ (Black et al., 1992), DESA1, acyl-carrier-protein S-malonyltransferase ( $f a b D)$

(Magnuson et al., 1992), $f a b F, f a b G$, enoyl-[acyl-carrier-protein] reductase ( $f a b I)$ (Bergler et al., 1996), $\beta$-hydroxyacyl-acyl carrier protein dehydratases (fabZ) (Heath \& Rock, 1996), acyl-lipid omega-6 desaturase (Delta-12 desaturase) (FAD2) (Kainou et al., 2006), acyl-ACP thioesterases $(F a t A / B)($ Salas \& Ohlrogge, 2002), and stearoyl-CoA desaturase (Delta-9 desaturase) (SCD) (Stukey, McDonough \& Martin, 1990) were clearly upregulated in Ch03 compared to Ch02. However, in Ch04 compared to Ch03 identified that out of the 24 previously mentioned unigenes, 13 genes encoding Accase, ACSL, fabD, fabF, fabG, fabI, and SCD were downregulated, while the 11 remaining genes were not differentially expressed. The above results indicated that there are more relevant DEGs in Ch02 vs Ch03 compared to Ch01 vs Ch02 and $\mathrm{Ch03}$ vs Ch04. Based on the observations of C. majus elaiosome development (Yang et al., 2015), the remarkable cell growth of the elaiosome also occurred during the flowering stage to the young seed stage (Ch02 to Ch03). Therefore, we inferred that the biosynthesis of saturated fatty acids and unsaturated fatty acids in C. majus elaisomes might primarily occur during this period.

\section{Identification of unigenes related to the biosynthesis of saturated fatty acid}

In the fatty acid biosynthesis metabolic pathway, a comparison of the DEGs in Ch02 compared to Ch01 (Table 2, File S6A) showed that most genes that are primarily involved in fatty acid biosynthesis were not significantly expressed during the flowering stage (Ch02), and only a few genes were upregulated. By contrast, the gene expression analysis in Ch03 compared to Ch02 (Table 2, File S6B) revealed that the majority of genes were significantly expressed during the 
325 326 327 328

young seed stage (Ch03). Most of these expressed genes, which encode Accase, ACSL, DESA1, $f a b D, f a b F, f a b G, f a b I, f a b Z$, and $F a t A / B$, were significantly upregulated in Ch03. On the other hand, a gene expression analysis in Ch04 compared to Ch03 (Table 2, File S6C) revealed that almost all genes were significantly downregulated during the mature seed stage (Ch04). The above results indicated that the biosynthesis of saturated fatty acid in C. majus elaisomes and seeds also mainly occurred during the flowering stage to the young seed stage. However, some unigenes that encode $F a b G$, exhibited inverse expression patterns. The above-mentioned genes were found to be downregulated in $\mathrm{Ch} 03$ compared to $\mathrm{Ch} 02$ but were upregulated in $\mathrm{Ch} 02$ compared to $\mathrm{Ch} 01$ and in $\mathrm{Ch} 04$ compared to $\mathrm{Ch} 03$ (Table 2). These genes are potentially involved in fatty acid synthesis through negative feedback signaling.

The enzymes FatA/B and DESAl, which are the key enzymes involved in palmitic acid synthesis (see File S6), are particularly noteworthy, because palmitic acid is the most abundant saturated fatty acid in C. majus elaisomes but is present in very low quantities in the corresponding seeds. FatA/B was slightly upregulated in $\mathrm{Ch} 02$ compared to $\mathrm{Ch} 01$ and significantly upregulated in $\mathrm{Ch} 03$ compared to $\mathrm{Ch} 02$, but was significantly downregulated in Ch04 compared to Ch03. DESA1 was significantly upregulated in both $\mathrm{Ch} 02$ compared to $\mathrm{Ch} 01$ and $\mathrm{Ch} 03$ compared to $\mathrm{Ch} 02$, but significantly downregulated in Ch04 compared to Ch03. The above findings indicate that the biosynthesis of palmitic acid in C. majus elaiosomes occurs from the flower bud stage to the flowering stage ( $\mathrm{Ch} 01$ to $\mathrm{Ch} 02$ ) but primarily occurs from the flowering stage to the young seed stage. Hughes et al. (1994) compared the fatty acid compositions of 12 plant species of elaiosomes and seven orders of insects and showed that palmitic acid levels in elaiosomes were particularly similar to those in insects. However, because C. majus was not included in Hughes's study, whether the high palmitic acid content in C. majus elaiosomes is similar to that in the ants that disperse the C. majus seeds remains unknown and needs further study.

\section{Identification of unigenes related to the biosynthesis of unsaturated fatty acid}

Figure 8 and Figure 9 show that significantly upregulated genes were identified in $\mathrm{Ch} 03$ compared to $\mathrm{Ch} 02$ and were involved in three processes in the biosynthesis of unsaturated fatty acid metabolic pathway. The first process is the conversion of palmitic acid (C16:0) to stearic acid (C18:0), which involves genes encoding the enzyme, $f a b G$. The second process is the 
355

356

357

358

359

360

361

362

363

364

365

366

367

368

369

370

371

372

373

374

375

376

377

378

379

380

381

382

383

conversion of stearic acid (C18:0) to oleic acid $(\mathrm{C} 18: 1, \triangle 9)$, which involves genes encoding the enzymes, $S C D$ (Delta-9 desaturase) and DESA1. The third process is the conversion of oleic acid $(\mathrm{C} 18: 1, \Delta 9)$ to linoleic acid $(\mathrm{C} 18: 2, \triangle 9 \Delta 12)$, which involves genes encoding the enzyme, FAD2 (Delta-12 desaturase). The above findings showed that some palmitic acid was converted into stearic acid (saturated fatty acid), and the latter was subsequently desaturated to form oleic acid and linoleic acid.

Linoleic acid is a poly-unsaturated fatty acid that cannot be synthesized by insects de novo and is thus required to be incorporated in their diet (Dadd, 1973). Previous studies have suggested increasing linoleic acid levels by stimulating the diaspore-carrying behavior of the ants (Gammans, Bullock \& Schönrogge, 2005). The high linoleic acid content in both elaisomes and seeds of C. majus indicated that the whole diaspores (seed + elaiosome) were probably used to attract ants that contain high levels of (essential) nutrients. In addition, oleic acid was shown to promote ant removal behavior (Marshall, Beattie \& Bollenbacher, 1979; Skidmore \& Heithaus, 1988; Brew, O'Dowd \& Rae, 1989; Hughes, Westoby \& Jurado, 1994). The higher oleic acid content in C. majus elaiosomes and the lower oleic acid content in their corresponding seeds suggested that oleic acid in C. majus might act as the primary stimulating substance to induce the collection of its diaspores by ants, as previously reported in other plant species.

\section{Validation of unigenes by quantitative real-time PCR (qRT-PCR)}

To validate the reliability of the RNA-seq results, four unigenes encoding $A C S L, D E S A 1, f a b G$, and FatB were randomly selected for qRT-PCR validation at the four different developmental stages. These four genes were all related to the fatty acid biosynthesis metabolic pathway and biosynthesis of unsaturated fatty acid metabolic pathway. The qRT-PCR results (File S7) showed that the expression patterns of the four selected genes were very similar to those of RNA sequencing data in Ch03 compared to Ch02 and in Ch04 compared to Ch03 (Fig. 10). In Ch01 compared to $\mathrm{Ch} 02$, the expression pattern of DESAl by qRT-PCR was consistent with that from RNA sequencing (Fig. 10B), and $A C S L$, $f a b G$, and FatB showed slight differences (Figs. 10A, $10 \mathrm{C}, 10 \mathrm{D})$. It was acceptable that some differences in direct comparison between qRT-PCR and RNA-seq results would occur due to biases in the library preparation for RNA-seq, different normalization approaches, and other technical biases (Li et al., 2010; Wei, Chung \& Zhao, 
384 2011). qRT-PCR results of the selected genes were generally consistent with the RNA 385 sequencing results, indicating that the RNA-seq results were reliable.

\section{CONCLUSIONS}

387 Chelidonium majus is a well-known ant-dispersal plant with numerous elaiosome-bearing seeds.

388 In this study, we performed whole-transcriptome profiling of C. majus elaiosomes and seeds by

389 Illumina NGS sequencing, and used times series RNA-seq to study the expression patterns of 390 genes associated with fatty acid biosynthesis in them for the first time. A total of 63,064

391 unigenes were generated and 41 significantly DEGs involved in lipid metabolism pathways were 392 identified. By analysis and comparison of the DEGs at the four developmental stages of the 393 elaiosomes and seeds, we confirmed that the fatty acid biosynthesis in the elaiosome is consistent

394 395 396 397 398 399 400 401 402 403

404 405 406 407 408 409 410 411 with its cellular growth. The candidate genes involved in the biosynthesis of three primary fatty acids in elaiosomes and seeds were also identified. Our research not only provided important insights into fatty acid biosynthesis in C. majus elaiosomes and seeds, but also served as a genomic resource for future evolutionary studies that focus on the convergent evolution of myrmecochorus plants.

\section{ACKNOWLEDGEMENTS}

The authors would like to acknowledge Beijing Badaling National Forest Park for support with plant materials. We also thank our colleagues for discussions and advice on this study. We are also grateful to two anonymous reviewers for improving our manuscript.

\section{REFERENCES}

Arora D, Sharma AA. 2013. Review on phytochemical and pharmacological potential of genus Chelidonium. Pharmacognosy Journal 5(4):184-190.

Beattie AJ. 1985. The evolutionary ecology of ant-plant mutualisms. Cambridge University Press.

Bergler H, Fuchsbichler S, Högenauer G, Turnowsky F. 1996. The enoyl-[acyl-carrier-protein] reductase $(F a b I)$ of Escherichia coli, which catalyzes a key regulatory step in fatty acid biosynthesis, accepts $N A D H$ and $N A D P H$ as cofactors and is inhibited by palmitoyl-CoA. European Journal of Biochemistry 242(3):689-694. 
412

413

414

415

416

417

418

419

420

421

422

423

424

425

426

427

428

429

430

431

432

433

434

435

436

437

438

439

440

441

442

443

444

445

Bilder P, Lightle S, Bainbridge G, Ohren J, Finzel B, Sun F, Holley S, AI-Kassim L, Spessard C, MeInick M, Newcomer M, Waldrop GL. 2006. The structure of the carboxyltransferase component of acetyl-coA carboxylase reveals a zinc-binding motif unique to the bacterial enzyme. Biochemistry 45(6):1712-1722.

Black PN, DiRusso CC, Metzger AK, Heimert TL. 1992. Cloning, sequencing, and expression of the fadD gene of Escherichia coli encoding acyl coenzyme A synthetase. Journal of Biological Chemistry 267(35):25513-25520.

Bono JM, Heithaus ER. 2002. Sex ratios and the distribution of elaiosomes in colonies of the ant, Aphaenogaster rudis. Insectes Sociaux 49(4):320-325.

Boulay R, Coll-Toledano J, Cerdá X. 2006. Geographic variations in Helleborus foetidus elaiosome lipid composition: implications for dispersal by ants. Chemoecology 16(1):1-7.

Brew CR, O'Dowd DJ, Rae ID. 1989. Seed dispersal by ants: behaviour-releasing compounds in elaiosomes. Oecologia 80(4):490-497.

Chen G, Huang SZ, Chen SC, Chen YH, Liu X, Sun WB. 2016. Chemical composition of diaspores of the myrmecochorous plant Stemona tuberosa Lour. Biochemical Systematics and Ecology 64:31-37.

Ciccarelli D, Andreucci AC, Pagni AM, Garbari F. 2005. Structure and development of the elaiosome in Myrtus communis L.(Myrtaceae) seeds. Flora 200(4):326-331.

Conesa A, Götz S, García-Gómez JM, Terol J, Talón M, Robles M. 2005. Blast2GO: a universal tool for annotation, visualization and analysis in functional genomics research. Bioinformatics 21(18):3674-3676.

Dadd RH. 1973. Insect nutrition: current developments and metabolic implications. Annual Review of Entomology 18(1):381-420.

Fischer RC, Ölzant SM, Wanek W, Mayer V. 2005. The fate of Corydalis cava elaiosomes within an ant colony of Myrmica rubra: elaiosomes are preferentially fed to larvae. Insectes Sociaux 52(1):5562.

Fischer RC, Richter A, Hadacek F, Mayer V. 2008. Chemical differences between seeds and elaiosomes indicate an adaptation to nutritional needs of ants. Oecologia 155(3):539-547.

Gammans N, Bullock JM, Schönrogge K. 2005. Ant benefits in a seed dispersal mutualism. Oecologia 146(1):43-49.

Giladi I. 2006. Choosing benefits or partners: a review of the evidence for the evolution of myrmecochory. Oikos 112(3):481-492.

Gorb E, Gorb S. 2000. Effects of seed aggregation on the removal rates of elaiosome-bearing Chelidonium majus and Viola odourata seeds carried by Formica polyctena ants. Ecological Research 15(2): 187-192.

Peer] reviewing PDF | (2018:12:33189:1:1:NEW 9 Mar 2019) 
446

447

448

449

450

451

452

453

454

455

456

457

458

459

460

461

462

463

464

465

466

467

468

469

470

471

472

473

474

475

476

477

478

479

Grabherr MG, Haas BJ, Yassour M, Levin JZ, Thompson DA, Amit I, Adiconis X, Fan L, Raychowdhury R, Zeng Q, Chen Z, Mauceli E, Hacohen N, Gnirke A, Rhind N, Palma F, Birren BW, Nusbaum C, Lindblad-Toh K, Friedman N, Regev A. 2011. Full-length transcriptome assembly from RNA-Seq data without a reference genome. Nature Biotechnology 29:644-52.

Heath RJ, Rock CO. 1996. Roles of the $F a b A$ and $F a b Z \beta$-hydroxyacyl-acyl carrier protein dehydratases in Escherichia coli fatty acid biosynthesis. Journal of Biological Chemistry 271(44):27795-27801.

Hughes L, Westoby M. 1990. Removal rates of seeds adapted for dispersal by ants. Ecology 71(1):138148.

Hughes L, Westoby M. 1992. Effect of diaspore characteristics on removal of seeds adapted for dispersal by ants. Ecology 73(4):1300-1312.

Hughes L, Westoby M, Jurado E. 1994. Convergence of elaiosomes and insect prey: evidence from ant foraging behaviour and fatty acid composition. Functional Ecology 8(3):358-365.

Kachroo A, Shanklin J, Whittle E, Lapchyk L, Hildebrand D, Kachroo P. 2007. The Arabidopsis stearoyl-acyl carrier protein-desaturase family and the contribution of leaf isoforms to oleic acid synthesis. Plant Molecular Biology 63(2):257-271.

Kainou K, Kamisaka Y, Kimura K, Uemura H. 2006. Isolation of $\Delta 12$ and $\omega 3$-fatty acid desaturase genes from the yeast Kluyveromyces lactis and their heterologous expression to produce linoleic and $\alpha$-linolenic acids in Saccharomyces cerevisiae. Yeast 23(8):605-612.

Kanehisa M, Goto S. 2000. KEGG: kyoto encyclopedia of genes and genomes. Nucleic Acids Research 28(1):27-30.

Kusmenoglu S, Rockwood LL, Gretz MR. 1989. Fatty acids and diacylglycerols from elaiosomes of some ant-dispersed seeds. Phytochemistry 28(10):2601-2602.

Lanza J, Schmitt MA, Awad AB. 1992. Comparative chemistry of elaiosomes of three species of Trillium. Journal of Chemical Ecology 18(2):209-221.

Lengyel S, Aaron DG, Andrew ML, Jonathan DM, Robert RD. 2010. Convergent evolution of seed dispersal by ants, and phylogeny and biogeography in flowering plants: a global survey. Perspectives in Plant Ecology, Evolution and Systematics 12(1):43-55.

Li B, Dewey CN. 2011. RSEM: accurate transcript quantification from RNA-Seq data with or without a reference genome. BMC Bioinformatics 12:323.

Li B, Ruotti V, Stewart RM, Thomson JA, Dewey CN. 2010. RNA-Seq gene expression estimation with read mapping uncertainty. Bioinformatics 26:493.

Lisci M, Bianchini M, Pacini E. 1996. Structure and function of the elaiosome in some angiosperm species. Flora 191(2):131-141. 
480

481

482

483

484

485

486

487

488

489

490

491

492

493

494

495

496

497

498

499

500

501

502

503

504

505

506

507

508

509

510

511

512

Magnuson K, Carey MR, Cronan JE. 1995. The putative fabJ gene of Escherichia coli fatty acid synthesis is the fabF gene. Journal of Bacteriology 177(12):3593-3595.

Magnuson K, Oh W, Larson TJ, Cronan Jr JE. 1992. Cloning and nucleotide sequence of the $f a b D$ gene encoding malonyl coenzyme A-acyl carrier protein transacylase of Escherichia coli. FEBS Letters 299(3):262-266.

Maji AK, Banerji P. 2015. Chelidonium majus L. (Greater celandine)-a review on its phytochemical and therapeutic perspectives. International Journal of Herbal Medcine 3:10-27.

Mark S, Olesen JM. 1996. Importance of elaiosome size to removal of ant-dispersed seeds. Oecologia 107(1):95-101.

Marshall DL, Beattie AJ, Bollenbacher WE. 1979. Evidence for diglycerides as attractants in an antseed interaction. Journal of Chemical Ecology 5(3):335-344.

Morales MA, Heithaus ER. 1998. Food from seed-dispersal mutualism shifts sex ratios in colonies of the ant Aphaenogaster rudis. Ecology 79(2):734-739.

Morrone O, Vega AS, Maier M. 2000. Elaiosomes in Urochloa paucispicata (Poaceae: Panicoideae: Paniceae): anatomy and chemical composition. Flora 195(4):303-310.

Oaxaca-Castillo D, Andreoletti P, Vluggens A, Yu S, Veldhoven PP, Reddy JK, Cherkaoui-Malki M. 2007. Biochemical characterization of two functional human liver acyl-CoA oxidase isoforms 1a and $1 \mathrm{~b}$ encoded by a single gene. Biochemical and Biophysical Research Communications 360(2):314-319.

Oostermeijer JGB. 1989. Myrmecochory in Polygala vulgaris L., Luzula campestris (L.) DC. and Viola curtisii Forster in a Dutch dune area. Oecologia 78(3):302-311.

Reifenrath K, Becker C, Poethke HJ. 2012. Diaspore trait preferences of dispersing ants. Journal of Chemical Ecology 38(9):1093-1104.

Robinson MD, McCarthy DJ, Smyth GK. 2010. EdgeR: a Bioconductor package for differential expression analysis of digital gene expression data. Bioinformatics 26(1):139-140.

Salas JJ, Ohlrogge JB. 2002. Characterization of substrate specificity of plant FatA and FatB acyl-ACP thioesterases. Archives of Biochemistry and Biophysics 403(1):25-34.

Sernander R. 1906. Entwurf einer Monographie der europaeischen Myrmekochoren. Kungl Svenska Vetenskapsakadem Handl 41:1-407.

Servigne P, Detrain C. 2008. Ant-seed interactions: combined effects of ant and plant species on seed removal patterns. Insectes Sociaux 55(3):220-230.

Skidmore BA, Heithaus ER. 1988. Lipid cues for seed-carrying by ants in Hepatica americana. Journal of Chemical Ecology 14(12):2185-2196. 
513 Soukup VG, Holman RT. 1987. Fatty acids of seeds of North American pedicillate Trillium species.

514

515

516

517

518

519

520

521

522

523

524

525

526

527

528

529

530

531

532

533

534

535

536

537

538

539

540

541

542

543 Phytochemistry 26(4):1015-1018.

Stukey JE, McDonough VM, Martin CE. 1990. The OLE1 gene of Saccharomyces cerevisiae encodes the delta 9 fatty acid desaturase and can be functionally replaced by the rat stearoyl-CoA desaturase gene. Journal of Biological Chemistry 265(33):20144-20149.

Tatusov RL, Fedorova ND, Jackson JD, Jacobs AR, Kiryutin B, Koonin EV, Krylov DM, Mazumder R, Mekhedov SL, Nikolskaya AN, Rao BS, Smirnov S, Sverdlov AV, Vasudevan S, Wolf YI, Yin JJ, Natale DA. 2003. The COG database: an updated version includes eukaryotes. BMC Bioinformatics 4:41.

Toomey RE, Wakil SJ. 1966. Studies on the mechanism of fatty acid synthesis XV. Preparation and general properties of $\beta$-ketoacyl acyl carrier protein reductase from Escherichia coli. Biochimica et Biophysica Acta 116(2):189-197.

Unamba CIN, Nag A, Sharma RK. 2015. Next generation sequencing technologies: the doorway to the unexplored genomics of non-model plants. Frontiers in Plant Science 6:1074.

Van der Pijl L. 1982. Principles of dispersal. Springer: Berlin, Germany.

Wei Z, Chung LM, Zhao H. 2011. Bias detection and correction in RNA-sequencing data. BMC Bioinformatics 12:290.

Werker E. 1997. Seed anatomy. Gebruder Borntraeger Verlagsbuchhandlung, Stuttgart, Germany.

Xie C, Mao X, Huang J, Ding Y, Wu J, Dong S, Kong L, Gao G, Li CY, Wei L. 2011. KOBAS 2.0: a web server for annotation and identification of enriched pathways and diseases. Nucleic Acids Research 39(Web Server issue):316-322.

Yang S, Li JX, Xia XF, Zhao LC. 2015. Elaiosome development in Chelidonium L. seeds. Bulletin of Botanical Research 35(2):191-199.

\section{Table caption:}

Table 1. Raw data and clean data statistics of RNA sequencing.

${ }^{1} \mathrm{GC} \%$ : The total number of bases $\mathrm{G}$ and $\mathrm{C}$ as a percentage of the total bases number; ${ }^{2} \mathrm{Q} 20 \%$ :

The percentage of bases whose Phred value is greater than 20 accounts for the total bases;

${ }^{3} \mathrm{Q} 30 \%$ : The percentage of bases whose Phred value is greater than 30 accounts for the total bases. 
544

545 Table 2. DEGs in fatty acid biosynthesis metabolic pathway for different developmental stages.

546 'UR: Upgraded unigenes number, data in bracket represents significantly DEGs. ${ }^{2} \mathrm{DR}$ :

547 Downgraded uingenes number, data in bracket represents significantly DEGs.

548

549 Figure caption:

550

551 Figure 1. Chelidonium majus (A-H). (A) The naturally growing plants in Beijing Badaling

552 National Forest Park, China. (B) Flower buds (Ch01). (C) Pollinated flower (Ch02). (D) Fruits.

553 (E) Young seeds with elaiosomes (Ch03). (F) Mature seeds with elaiosomes (Ch04). (G)

554 Enlargement of mature seeds with elaiosomes, showing the white elaiosome is contrasted with

555 the glossy black seed. (H) Longitudinal section of mature seed and elaiosome, showing the red-

556 stained lipids in broken elaiosome and massive protein granulars in endosperm. (I) Diaspore

557 (seed + elaiosome) removal of $C$. majus by ant. Bars: $(\mathrm{A})=10 \mathrm{~cm},(\mathrm{~B}-\mathrm{D})=1 \mathrm{~cm},(\mathrm{E})=3 \mathrm{~mm}$,

$558(\mathrm{~F})=2 \mathrm{~mm},(\mathrm{G}, \mathrm{I})=1 \mathrm{~mm},(\mathrm{H})=100 \mu \mathrm{m}$. Abbreviation, EL: elaiosome, EN: endosperm.

559 (Photoed by Liangcheng Zhao)

560

561 Figure 2. Statistic of trinity assembly results. N50: Assembled unigenes by length from large to 562 small, and the length of it is half the length of the unigenes to the total (bp). N90: Assembled

563 unigenes by length from large to small, and the length of it is $90 \%$ the length of the unigenes to 564 the total (bp).

565

566 Figure 3. Characteristics of sequence homology of unigenes against the NR database: (A) E567 value distribution of BLAST hits for each unigene with an E-value cut-off of $1.0 \times 10^{-5}$. (B) 568 Similarity distribution of the top BLAST hits for each unigene. (C) Species distribution of 569 the top BLAST hits. 
570 Figure 4. GO classification of assembled unigenes. The abscissa represents the secondary 571 classification terms of GO, the left ordinate represents the percentage of unigenes contained in 572 the secondary classification, the right ordinate represents the number of unigenes. This three 573 colors represent three categories, of which green represents biological process, blue represents 574 cellular component and red represents molecular function.

575

576 Figure 5. COG function classification of assembled unigenes. Each column represents the 577 functional classification of $\mathrm{COG}$ (Capital letters $\mathrm{A} \sim \mathrm{Z}$ are used to indicate the specific meaning, 578 as indicated on the right), and the height of the column is the ordinate of the number of unigenes 579 with this function.

580

581 Figure 6. Distribution of unigenes in different KEGG pathways. Genes were divided into 5 582 clusters. A: Metabolism. B: Genetic Information Processing. C: Environmental Information 583 Processing. D: Cellular Process. E: Organismal Systems.

584

585 Figure 7. Pathways in 'lipid metabolism' group and number of the unigenes. Each column 586 represents the lipid metabolism pathways of KEGG, and the height of the column is the 587 ordinate of the number of unigenes with this pathway.

588

589 Figure 8. DEGs from the fatty acid biosynthesis and biosynthesis of unsaturated fatty acid 590 metabolism diagram. The locus tag and enzyme code shown in fatty acid biosynthesis and 591 biosynthesis of unsaturated fatty acid metabolism are described in a heat map.

592

593 Figure 9. The detail RNA degradation database in biosynthesis of unsaturated fatty acid 594 pathway. The red border genes are those that can annotate on these gene products. 
596 Figure 10. QRT-PCR quantification and RNA-seq results of four selected genes in different 597 developmental stages. (A) ACSL. (B) DESA1. (C) FabG. (D) FatB. 


\section{Figure 1}

Chelidonium majus.

(A) The naturally growing plants in Beijing Badaling National Forest Park, China. (B) Flower buds (Ch01). (C) Pollinated flower (Ch02). (D) Fruits. (E) Young seeds with elaiosomes (Ch03). (F) Mature seeds with elaiosomes (Ch04). (G) Enlargement of mature seeds with elaiosomes, showing the white elaiosome is contrasted with the glossy black seed. $(\mathbf{H})$ Longitudinal section of mature seed and elaiosome, showing the red-stained lipids in broken elaiosome and massive protein granulars in endosperm. (I) Diaspore (seed + elaiosome) removal of $C$. majus by ant. Bars: $(A)=10 \mathrm{~cm},(B-D)=1 \mathrm{~cm},(E)=3 \mathrm{~mm},(F)=2 \mathrm{~mm},(\mathrm{G}, \mathrm{I})$ $=1 \mathrm{~mm},(\mathrm{H})=100 \mu \mathrm{m}$. Abbreviation, EL: elaiosome, EN: endosperm. (Photoed by Liangcheng Zhao) 

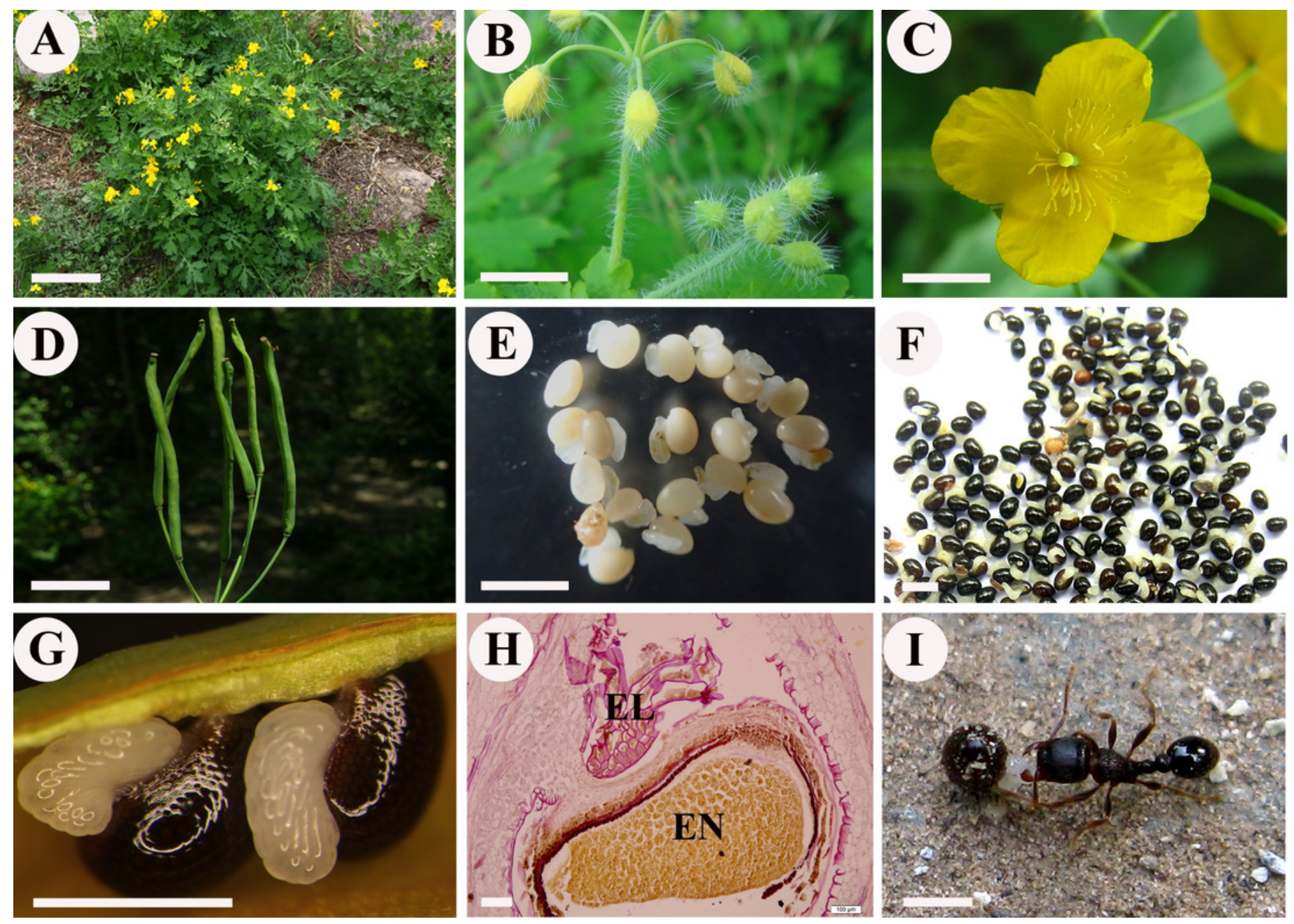
Figure 2 (on next page)

Statistic of trinity assembly results.

N50: Assembled unigenes by length from large to small, and the length of it is half the length of the unigenes to the total (bp). N90: Assembled unigenes by length from large to small, and the length of it is $90 \%$ the length of the unigenes to the total (bp). 


\section{Seq Length Distribution}

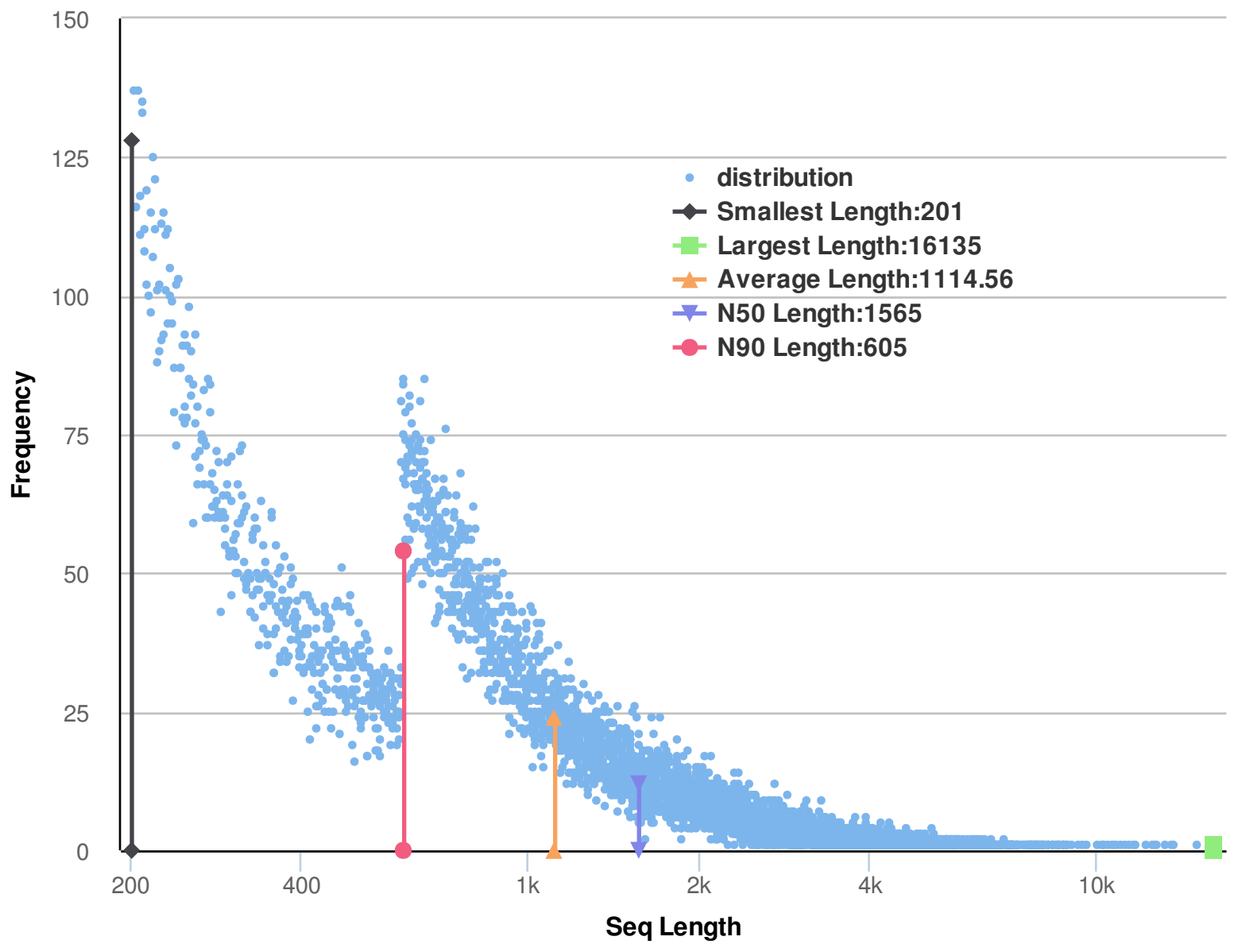




\section{Figure 3}

Characteristics of sequence homology of unigenes against NR database.

(A) E-value distribution of BLAST hits for each unigene with an E-value cut-off of $1.0 \times 10^{-5}$.

Similarity distribution of the top BLAST hits for each unigene. (C) Species distribution of the top BLAST hits.
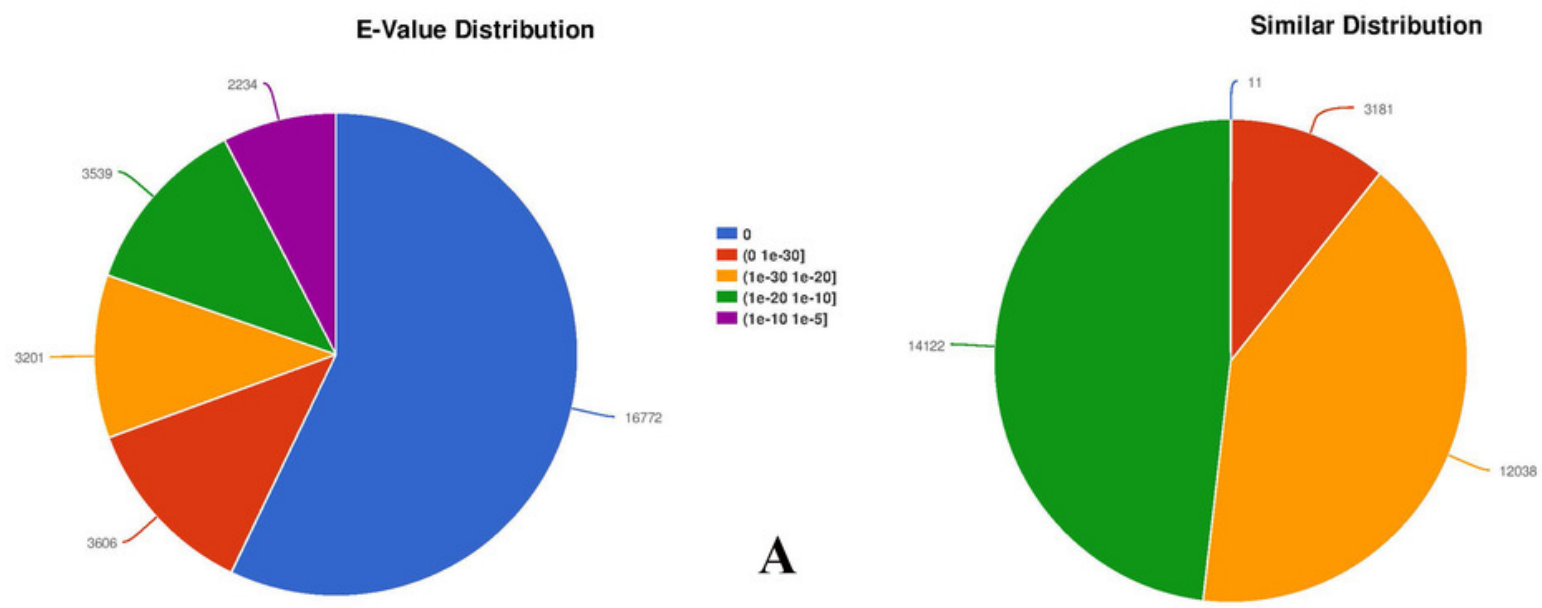

르 $20 \%$ to $40 \%$

$40 \%$ to $60 \%$

$60 \%$ to $80 \%$

$80 \%$ to $100 \%$

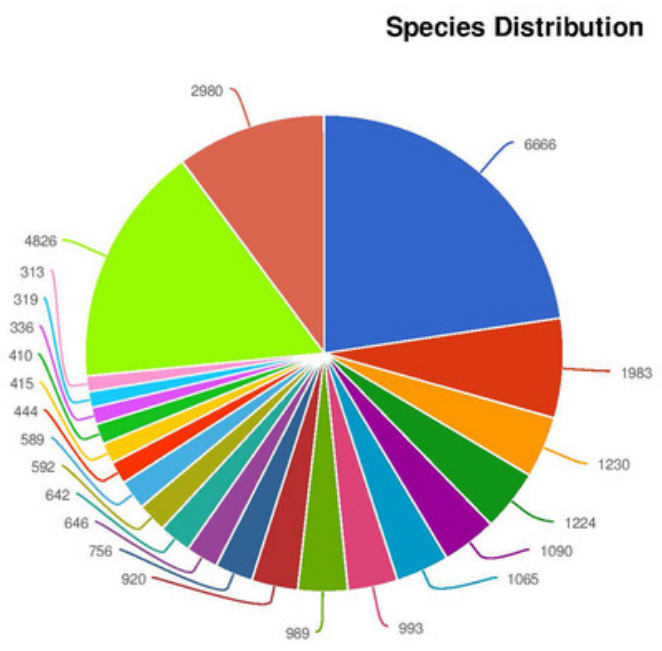

Vitis vinifera

- Theobroma cacao

Phoenix dactylifera

Populus trichocarp

- Prunus persica

Jatropha curcas

Malus domestica

Prunus mume

Citrus clementin

Morus notabills

Citrus sinensis

Eucalyptus grandis

Coffea canephora

- Danio rerio

- Glycine max

Eragaria vesca

Erythranthe guttat

- Cucumis melo

Solanum lycopersicum

=

species unknown

B

\section{C}


Figure 4 (on next page)

GO classification of assembled unigenes.

The abscissa represents the secondary classification terms of $\mathrm{GO}$, the left ordinate represents the percentage of unigenes contained in the secondary classification, the right ordinate represents the number of unigenes. This three colors represent three categories, of which green represents biological process, blue represents cellular component and red represents molecular function. 


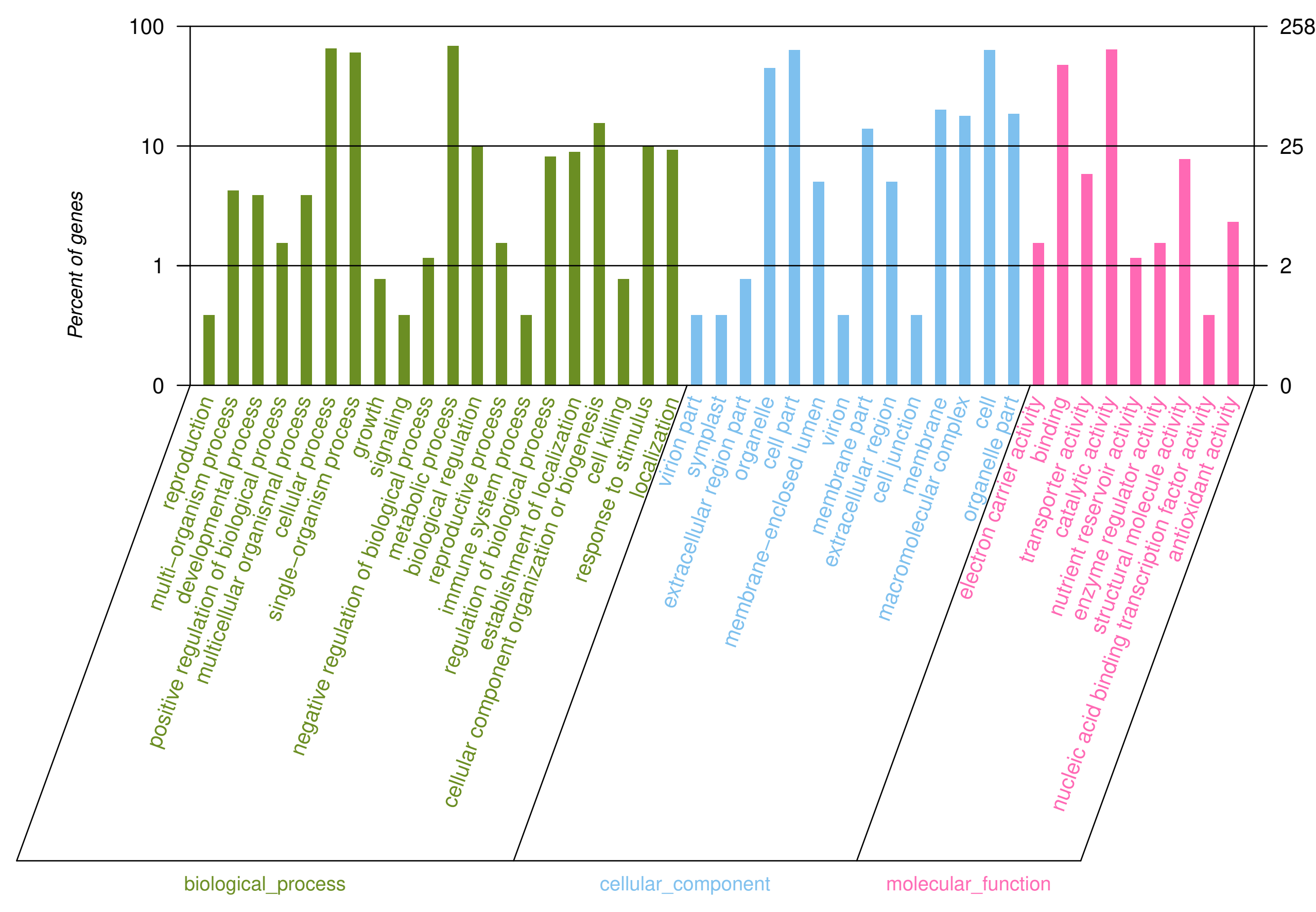


Figure $\mathbf{5}$ (on next page)

COG function classification of assembled unigenes.

Each column represents the functional classification of COG (Capital letters A Z are used to indicate the specific meaning, as indicated on the right), and the height of the column is the ordinate of the number of unigenes with this function. 


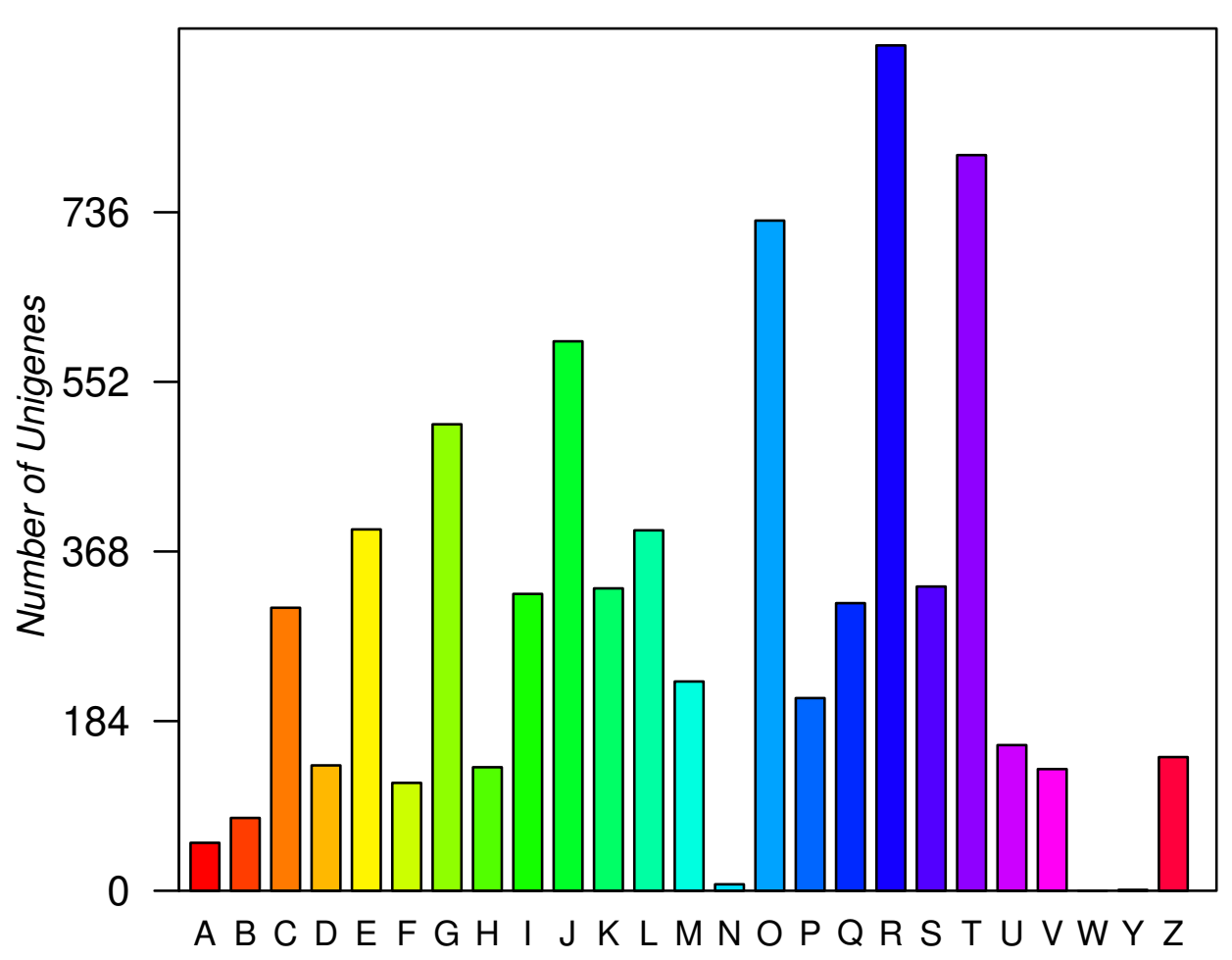

Function Class
A : RNA processing and modification

$B$ : Chromatin structure and dynamics

C : Energy production and conversion

D : Cell cycle control, cell division, chromosome partitioning

E : Amino acid transport and metabolism

$F$ : Nucleotide transport and metabolism

G : Carbohydrate transport and metabolism

$\mathrm{H}$ : Coenzyme transport and metabolism

I : Lipid transport and metabolism

$\mathrm{J}$ : Translation, ribosomal structure and biogenesis

K : Transcription

$\mathrm{L}$ : Replication, recombination and repair

$\mathrm{M}$ : Cell wall/membrane/envelope biogenesis

$\mathrm{N}$ : Cell motility

O : Posttranslational modification, protein turnover, chaperones

$\mathrm{P}$ : Inorganic ion transport and metabolism

Q: Secondary metabolites biosynthesis, transport and catabolism

$R$ : General function prediction only

$S$ : Function unknown

$\mathrm{T}$ : Signal transduction mechanisms

$\mathrm{U}$ : Intracellular trafficking, secretion, and vesicular transport

$\mathrm{V}$ : Defense mechanisms

$W$ : Extracellular structures

$\mathrm{Y}$ : Nuclear structure

Z : Cytoskeleton 
Figure 6 (on next page)

Distribution of unigenes in different KEGG pathways.

Genes were divided into 5 clusters. A: Metabolism. B: Genetic Information Processing. C:

Environmental Information Processing. D: Cellular Process. E: Organismal Systems. 


\section{Sensory system $\square 145$ \\ Nervous system $\square 190$ \\ Immune system \\ Excretory system $\square 85$ \\ Environmental adaptation $\square 350$ \\ Endocrine system 453 \\ Digestive system $\square 210$ \\ Development $\square 78$ \\ Circulatory system $\square 100$ \\ Transport and catabolism
Cell motility 45 \\ Cell growth and death \\ Cell communication $\square 145$}

Signaling molecules and interaction $\square 288$

Signal transduction

Membrane transport

Translation

Transcription $\square 107$

Replication and repair

Folding, sorting and degradation

Xenobiotics biodegradation and metabolism $\square 117$

Nucleotide metabolism

Metabolism of terpenoids and polyketides

Metabolism of other amino acids

Metabolism of cofactors and vitamins

Lipid metabolism

Glycan biosynthesis and metabolism

Global and overview maps

Energy metabolism

Chemical structure transformation maps 33

Carbohydrate metabolism

Biosynthesis of other secondary metabolites

Amino acid metabolism
878

1099

\begin{tabular}{|c|c|c|c|c|c|c|c|c|}
\hline 0 & 5 & 10 & 15 & 20 & 25 & 30 & 35 & 40 \\
\hline
\end{tabular}




\section{Figure 7}

Pathways in 'lipid metabolism' group and number of their unigenes.

Each column represents the lipid metabolism pathways of KEGG, and the height of the column is the ordinate of the number of unigenes with this pathway.

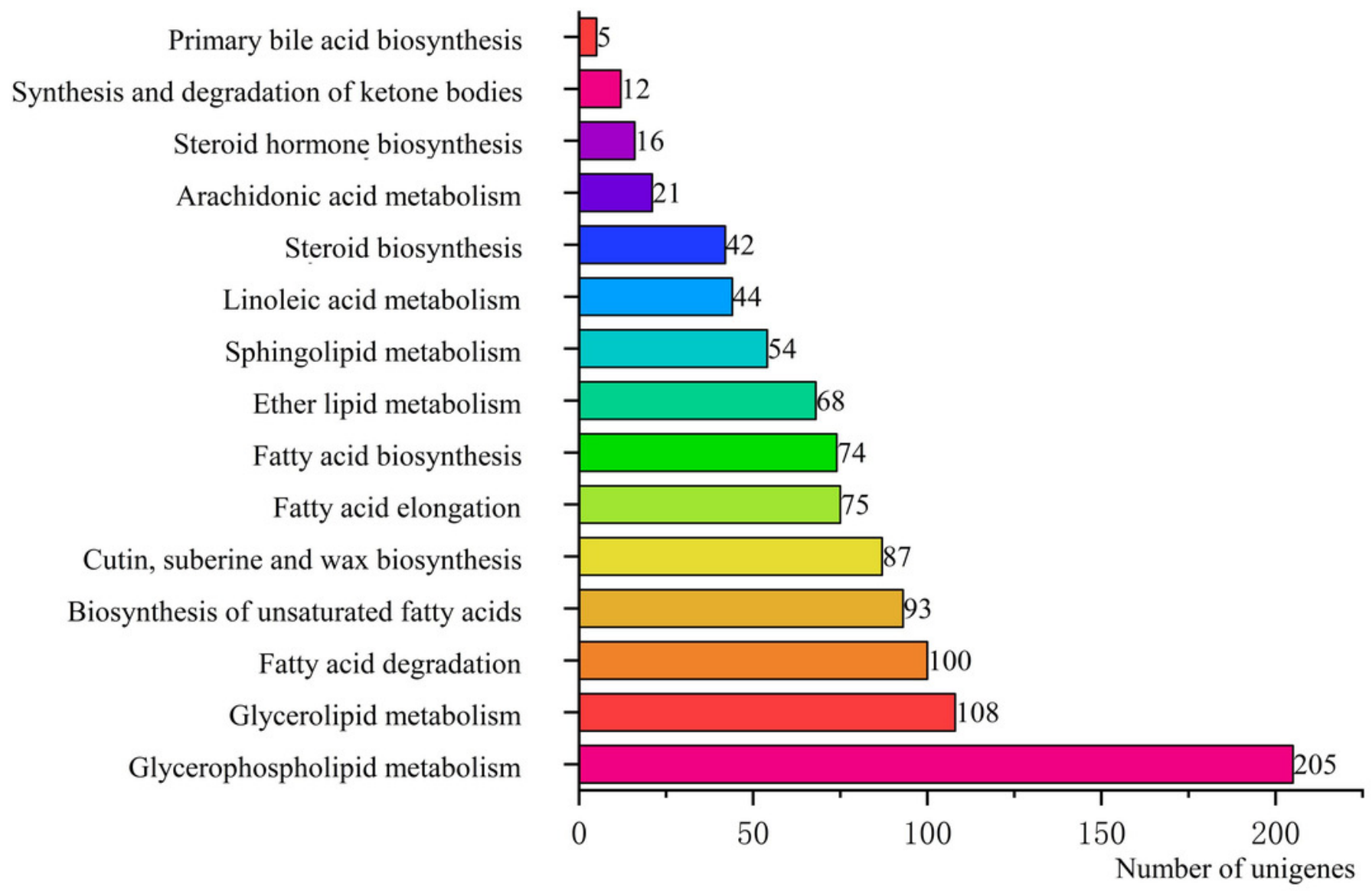


Figure 8 (on next page)

DEGs from the fatty acid biosynthesis pathway and biosynthesis of unsaturated fatty acid pathway diagram.

The locus tag and enzyme code shown in fatty acid biosynthesis pathway and biosynthesis of unsaturated fatty acid pathway are described in a heat map. 
Figure 9

The detail RNA degradation database in biosynthesis of unsaturated fatty acid pathway.

The red border are those genes that can annotate on these gene products.

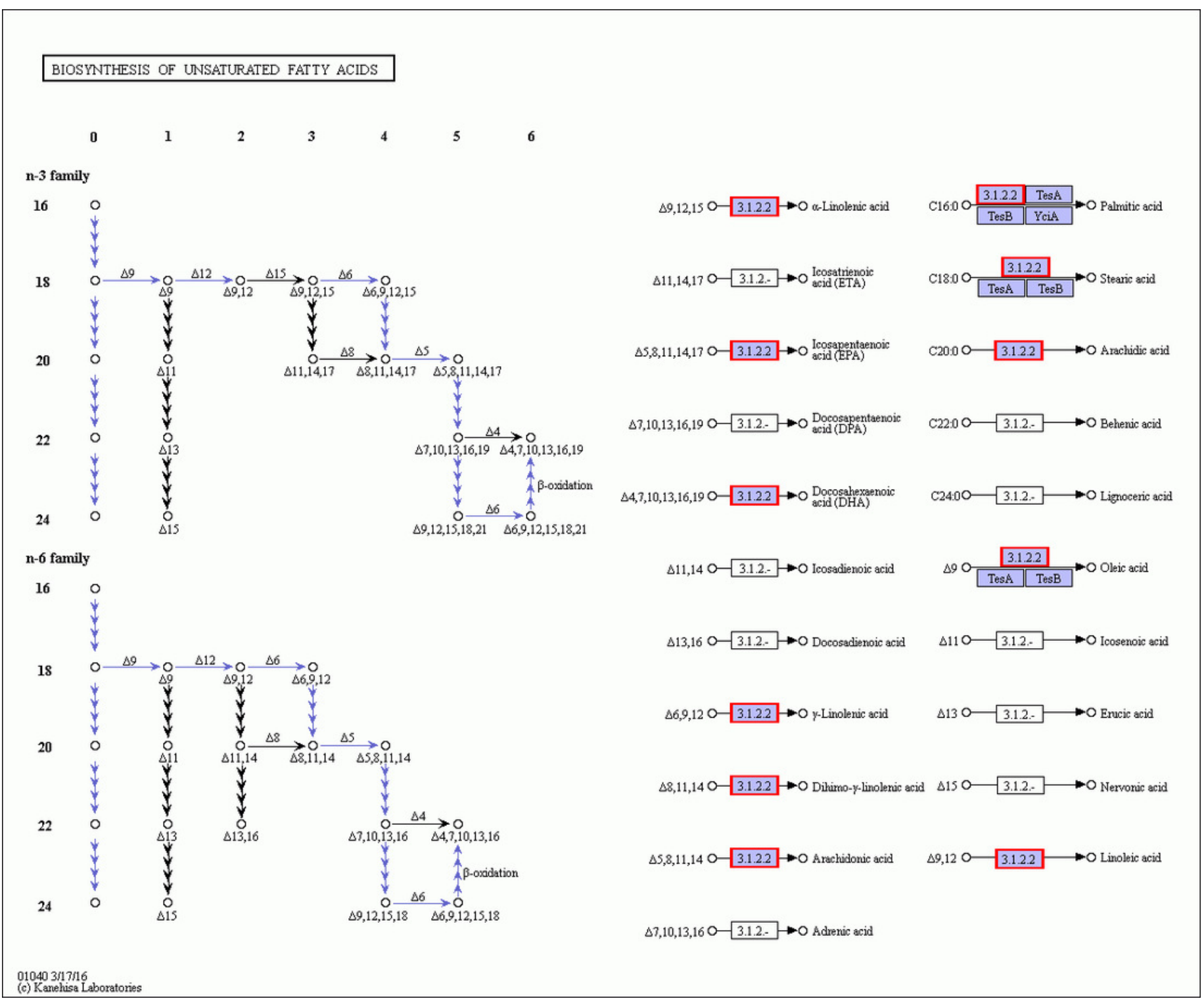


Figure 10 (on next page)

qRT-PCR quantification and RNA-seq results of four selected genes in different developmental stages of $C$. majus .
(A) ACSL. (B) DESA1. (C) FabG. (D) FatB. 
PeerJ
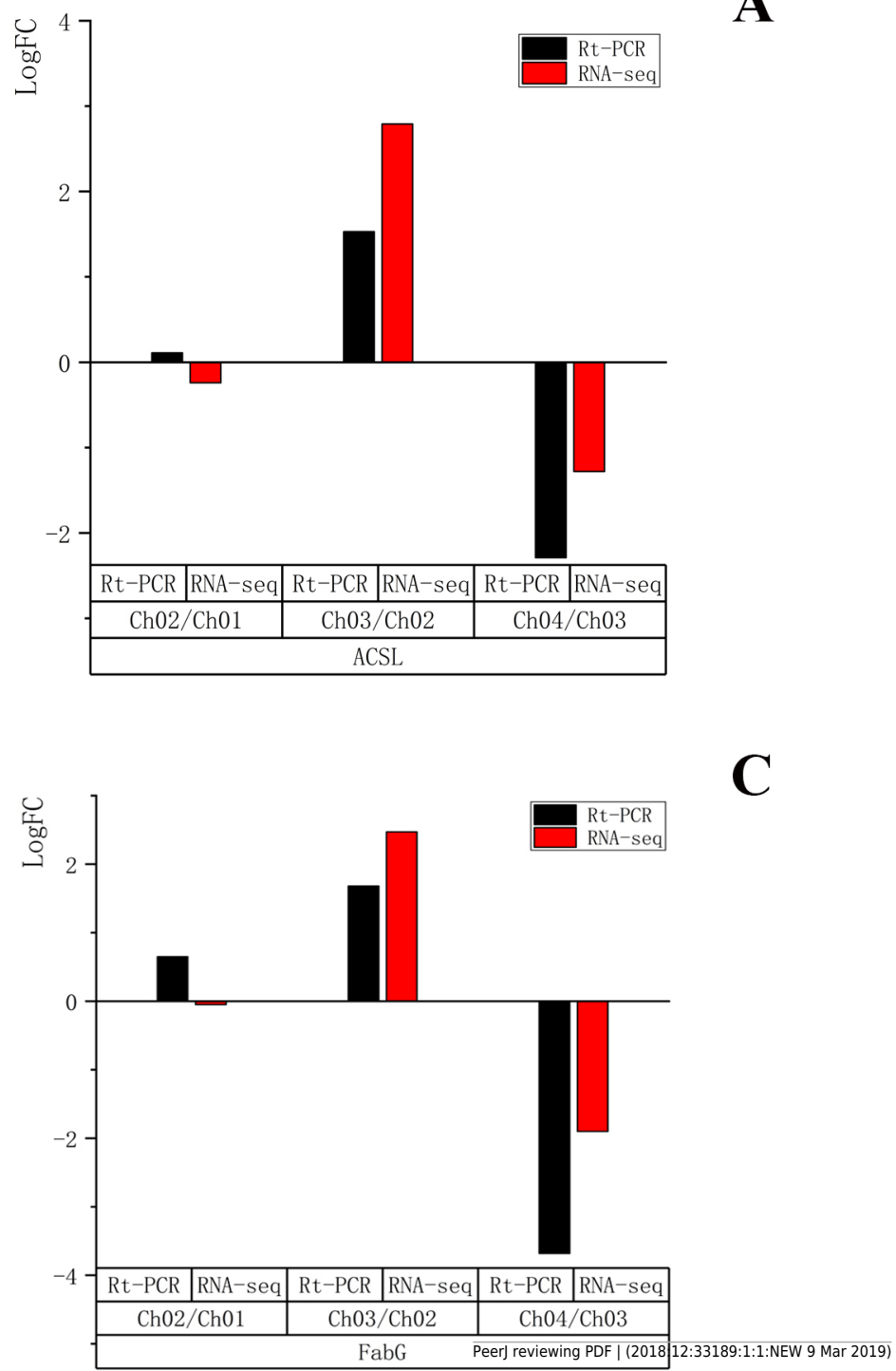

A
Manuscript to be reviewed

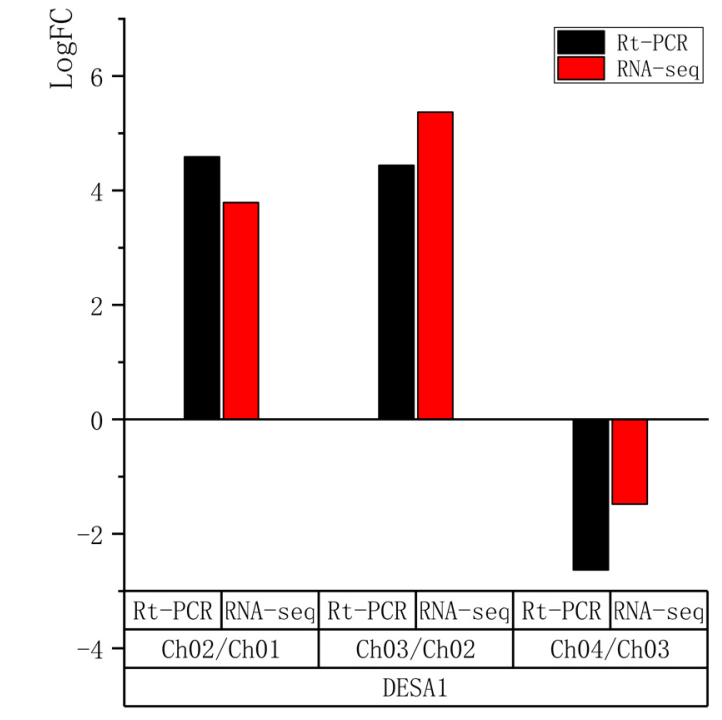

B

D

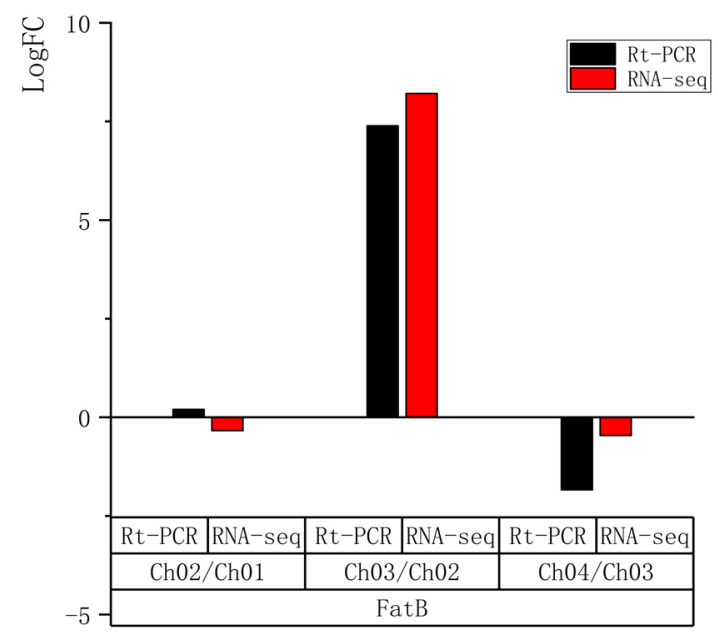




\section{Table 1 (on next page)}

Raw data and valid data statistics of RNA sequencing.

${ }^{1} \mathrm{GC} \%$ : The total number of bases $\mathrm{G}$ and $\mathrm{C}$ as a percentage of the total bases number; ${ }^{2} \mathrm{Q} 20 \%$ : The percentage of bases whose Phred value is greater than 20 accounts for the total bases; ${ }^{3} \mathrm{Q} 30 \%$ : The percentage of bases whose Phred value is greater than 30 accounts for the total bases. 
Table 1. Raw data and clean data statistics of RNA sequencing.

\begin{tabular}{|c|c|c|c|c|c|c|c|}
\hline Sample_ID & Raw_Reads & Clean_Reads & Clean_Bases & Error\% & $\mathbf{Q 2 0 \%}{ }^{2}$ & Q30\% ${ }^{3}$ & $\mathrm{GC}^{1}{ }^{1}$ \\
\hline Ch01a & $46,184,952$ & $44,177,128$ & $6,461,240,190$ & 0.0138 & 97.66 & 93.3 & 45.02 \\
\hline Ch01b & $56,008,592$ & $54,268,742$ & $7,954,613,129$ & 0.0135 & 97.8 & 93.66 & 42.33 \\
\hline Ch01c & $75,452,800$ & $72,811,640$ & $10,706,410,254$ & 0.0136 & 97.78 & 93.53 & 42.81 \\
\hline Ch01（total） & $177,646,344$ & $171,257,510$ & $25,122,263,573$ & 0.0136 & 97.75 & 93.5 & 43.39 \\
\hline $\mathrm{Ch} 02 \mathrm{a}$ & $49,629,792$ & $47,787,888$ & $7,035,686,679$ & 0.0135 & 97.81 & 93.63 & 43.52 \\
\hline $\mathrm{Ch} 02 \mathrm{~b}$ & $48,985,314$ & $46,706,162$ & $6,845,643,793$ & 0.014 & 97.58 & 93.13 & 44.03 \\
\hline $\mathrm{Ch} 02 \mathrm{c}$ & $48,604,760$ & $46,676,484$ & $6,849,388,718$ & 0.0137 & 97.72 & 93.43 & 44.32 \\
\hline Ch02（total） & $147,219,866$ & $141,170,534$ & $20,730,719,190$ & 0.0137 & 90.7 & 93.4 & 43.95 \\
\hline Ch03a & $46,603,464$ & $45,034,614$ & $6,637,326,310$ & 0.0137 & 97.76 & 93.5 & 43.75 \\
\hline $\mathrm{Ch} 03 \mathrm{~b}$ & $51,901,360$ & $50,226,988$ & $7,404,348,166$ & 0.0135 & 97.83 & 93.67 & 43.56 \\
\hline Ch03c & $53,968,514$ & $52,416,848$ & $7,726,545,508$ & 0.0133 & 97.92 & 93.88 & 43.54 \\
\hline Ch03（total） & $152,473,338$ & $147,678,450$ & $21,768,219,984$ & 0.0135 & 97.84 & 93.68 & 43.62 \\
\hline Ch04a & $52,126,852$ & $50,435,348$ & $7,426,968,007$ & 0.0135 & 97.84 & 93.67 & 43.67 \\
\hline $\mathrm{Ch} 04 \mathrm{~b}$ & $53,366,564$ & $51,854,584$ & $7,647,296,324$ & 0.0133 & 97.92 & 93.86 & 43.7 \\
\hline $\mathrm{Ch04c}$ & $52,973,818$ & $51,264,748$ & $7,548,125,708$ & 0.0135 & 97.84 & 93.72 & 43.48 \\
\hline Ch04（total） & $158,467,234$ & $153,554,680$ & $22,622,390,039$ & 0.0134 & 97.87 & 93.75 & 43.62 \\
\hline
\end{tabular}

$2 \quad{ }^{1} \mathrm{GC} \%$ : The total number of bases $\mathrm{G}$ and $\mathrm{C}$ as a percentage of the total bases number; ${ }^{2} \mathrm{Q} 20 \%$ : The percentage of bases whose

3 Phred value is greater than 20 accounts for the total bases; ${ }^{3} \mathrm{Q} 30 \%$ : The percentage of bases whose Phred value is greater than 30 4 accounts for the total bases. 


\section{Table 2 (on next page)}

DEGs in fatty acid biosynthesis metabolic pathway for different developmental stages.

${ }^{1}$ UR: Upgraded unigenes number, data in bracket represents significantly DEGs. ${ }^{2} D R$ :

Downgraded uingenes number, data in bracket represents significantly DEGs. 
Table 2. DEGs in fatty acid biosynthesis metabolic pathway for different developmental stages.

\begin{tabular}{|c|c|c|c|c|c|c|c|c|c|c|}
\hline \multirow{3}{*}{$\begin{array}{l}\text { KEGG } \\
\text { pathway }\end{array}$} & \multirow{3}{*}{ Gene } & \multirow{3}{*}{$\begin{array}{l}\text { Gene } \\
\text { ID }\end{array}$} & \multirow{3}{*}{ Gene name } & \multirow{3}{*}{ Enzyme } & \multirow{2}{*}{\multicolumn{2}{|c|}{$\begin{array}{l}\text { Ch01 vs Ch02 } \\
\text { DEGs number }\end{array}$}} & \multirow{2}{*}{\multicolumn{2}{|c|}{$\begin{array}{l}\text { Ch02 vs Ch03 } \\
\text { DEGs number }\end{array}$}} & \multirow{2}{*}{\multicolumn{2}{|c|}{$\begin{array}{l}\text { Ch03 vs Ch04 } \\
\text { DEGs number }\end{array}$}} \\
\hline & & & & & & & & & & \\
\hline & & & & & $\mathbf{U R}^{1}$ & $\mathbf{D R}^{2}$ & UR & DR & UR & DR \\
\hline path:ko00061 & Accase (accA) & K01962 & acetyl-CoA carboxylase & EC 6.4.1.2 & 0 & $2(0)$ & 1(1) & $1(0)$ & $1(0)$ & 1(1) \\
\hline path:ko00061 & Accase (accB) & K02160 & acetyl-CoA carboxylase & EC 6.4.1.2 & $2(0)$ & $1(0)$ & $3(3)$ & 0 & $2(0)$ & 1(1) \\
\hline path:ko00061 & Accase $(\operatorname{accC})$ & K01961 & acetyl-CoA carboxylase & EC 6.4.1.2 & 0 & $1(0)$ & 1(1) & 0 & 0 & 1(1) \\
\hline path:ko00061 & Accase (accD) & K01963 & acetyl-CoA carboxylase & EC 6.4.1.2 & $1(0)$ & 0 & 1(1) & 0 & 0 & 1(1) \\
\hline path:ko00061 & Accase (ACACA) & K11262 & acetyl-CoA carboxylase & EC 6.4.1.2 & $1(0)$ & 0 & 0 & $1(0)$ & $1(0)$ & 0 \\
\hline path:ko00061 & fabD & K00645 & [acyl-carrier-protein] S-malonyltransferase & EC 2.3.1.39 & 0 & $1(0)$ & $1(1)$ & 0 & 0 & 1(1) \\
\hline path:ko00061 & fabF & K09458 & 3-oxoacyl-[acyl-carrier-protein] synthase II & EC 2.3.1.179 & $1(1)$ & $4(0)$ & $5(4)$ & 0 & $1(0)$ & 4(3) \\
\hline path:ko00061 & fabG & K00059 & beta-ketoacyl reductase & EC 1.1.1.100 & $1(1)$ & $2(0)$ & $2(1)$ & $1(1)$ & 2(1) & 1(1) \\
\hline path:ko00061 & DESA1 & K03921 & acyl carrier protein desaturase & EC 1.14.19.2 & $1(1)$ & $1(0)$ & $2(2)$ & 0 & 0 & $2(0)$ \\
\hline path:ko00061 & fabZ & K02372 & $\beta$-hydroxyacyl-acyl carrier protein dehydratases & EC 4.2.1.59 & $1(0)$ & 0 & 1(1) & 0 & 0 & $1(0)$ \\
\hline path:ko00061 & fabI & K00208 & enoyl-[acyl-carrier-protein] reductase & EC 1.3.1.10 & 0 & $1(0)$ & 1(1) & 0 & 0 & 1(1) \\
\hline path:ko00061 & FATA & K10782 & acyl-ACP thioesterase A & EC 3.1.2.14 & 0 & $1(0)$ & $1(1)$ & 0 & 0 & $1(0)$ \\
\hline path:ko00061 & FATB & K10781 & acyl-ACP thioesterase B & EC 3.1.2.14 & $1(0)$ & $2(0)$ & $2(1)$ & 0 & $2(1)$ & $1(0)$ \\
\hline path:ko00061 & ACSL & K01897 & acyl coenzyme A synthetase & EC 6.2.1.3 & $4(0)$ & $4(0)$ & $7(1)$ & $1(0)$ & $5(2)$ & $3(2)$ \\
\hline
\end{tabular}


$2{ }^{1}$ UR: Upgraded unigenes number, data in bracket represents significantly DEGs. ${ }^{2}$ DR: Downgraded uingenes number, data in bracket represents significantly DEGs. 